Acta Crystallographica Section D

\section{Biological Crystallography}

ISSN 1399-0047

Christina M. Cuttitta, ${ }^{\mathrm{a}, \mathrm{b}} \neq$ Daniel L. Ericson, ${ }^{\mathrm{a}, \mathrm{c}} \neq$ Alexander Scalia, ${ }^{\mathrm{a}, \mathrm{d}} \neq$ Christian G. Roessler, ${ }^{{ }^{e}} \neq$ Ella Teplitsky, ${ }^{a, f} \neq$ Karan Joshi, ${ }^{\mathrm{a}, \mathrm{g}} \neq$ Olven Campos, ${ }^{\text {a,h }}$ Rakhi Agarwal,' Marc Allaire, ${ }^{\mathrm{e}}$ Allen M. Orville, ${ }^{\mathrm{e}, \mathrm{i}}$ Robert M. Sweet ${ }^{\mathrm{e}}$ and Alexei S. Soares ${ }^{\mathrm{e}}$ *

${ }^{a}$ Office of Educational Programs, Brookhaven National Laboratory, Upton, NY 11973-5000, USA, ${ }^{\mathbf{b}}$ Center for Developmental Neuroscience and Department of Biology, College of Staten Island, The City University of New York, 2800 Victory Boulevard, Staten Island, NY 10314, USA, 'Department of Biomedical Engineering, University at Buffalo, SUNY, 12 Capen Hall, Buffalo, NY 14260, USA, dDepartment of Biological Sciences, Binghamton University, 4400 Vestal Parkway East, Binghamton, NY 11973-5000, USA, ${ }^{\mathbf{e} P h o t o n ~ S c i e n c e s ~}$ Directorate, Brookhaven National Laboratory, Upton, NY 11973-5000, USA, 'Department of Biochemistry and Cell Biology, Stony Brook University, Stony Brook, NY 11794-5215, USA, ${ }^{\mathrm{g}}$ Department of Electronics and Electrical Communication Engineering, PEC University of Technology, Chandigarh, India, ' Department of Biological Science, Florida Atlantic University,

777 Glades Road, Boca Raton, FL 33414, USA, and 'Biosciences Department, Brookhaven National Laboratory, Upton, NY 11973-5000, USA

₹ These authors contributed equally to this work.

Correspondence e-mail: soares@bnl.gov

\title{
Acoustic transfer of protein crystals from agarose pedestals to micromeshes for high-throughput screening
}

Acoustic droplet ejection (ADE) is an emerging technology with broad applications in serial crystallography such as growing, improving and manipulating protein crystals. One application of this technology is to gently transfer crystals onto MiTeGen micromeshes with minimal solvent. Once mounted on a micromesh, each crystal can be combined with different chemicals such as crystal-improving additives or a fragment library. Acoustic crystal mounting is fast $\left(2.33\right.$ transfers s $\left.^{-1}\right)$ and all transfers occur in a sealed environment that is in vapor equilibrium with the mother liquor. Here, a system is presented to retain crystals near the ejection point and away from the inaccessible dead volume at the bottom of the well by placing the crystals on a concave agarose pedestal (CAP) with the same chemical composition as the crystal mother liquor. The bowl-shaped CAP is impenetrable to crystals. Consequently, gravity will gently move the crystals into the optimal location for acoustic ejection. It is demonstrated that an agarose pedestal of this type is compatible with most commercially available crystallization conditions and that protein crystals are readily transferred from the agarose pedestal onto micromeshes with no loss in diffraction quality. It is also shown that crystals can be grown directly on CAPs, which avoids the need to transfer the crystals from the hanging drop to a CAP. This technology has been used to combine thermolysin and lysozyme crystals with an assortment of anomalously scattering heavy atoms. The results point towards a fast nanolitre method for crystal mounting and highthroughput screening.

\section{Introduction}

Acoustic droplet ejection (ADE) is an automated and keyboard-driven technology for growing protein crystals (Yin et al., 2014; Villaseñor et al., 2012), improving the quality of protein crystals (Villaseñor et al., 2010) and transferring protein crystals onto data-collection media (Soares et al., 2011) such as pin-mounted micromesh sample holders. ADE transfers momentum from a sound pulse to move liquids and suspended crystals from the source location through a short air column to an arbitrary destination (Ellson et al., 2003; Fig. 1) with a trajectory precision of $\sim 1.3^{\circ}$ for solutions of $\sim 30 \mu \mathrm{m}$ crystals.

High-throughput screening of chemical libraries (such as fragment libraries) using X-ray crystallography requires a fast and flexible crystal-mounting technology. Acoustic crystal mounting is an attractive choice for high-throughput screening applications (Table 1). Since ADE is automated, its success is not dependent on the manual dexterity or physical aptitude of
Received 10 February 2014 Accepted 12 June 2014 
Table 1

Characteristics of different crystal-harvesting techniques.

We used acoustic methods to mount crystals on micromeshes and then to add chemicals that soak into the already mounted crystals [Le Maire et al. (2011) refer to soaking chemicals with crystals that were ready for data collection on plates as in crystallo soaking]. Characteristics of robotic crystal-harvesting techniques such as the universal manipulation robot (UMR) are shown in the column headed 'Robotic' (Viola et al., 2011) and manual crystal-harvesting characteristics are shown in the column headed 'Hand mount'. The time needed to mount crystals was measured for acoustic mounting with the Echo 550 (see §). The transfer speeds using other techniques were obtained from published videos (http:// www.ruppweb.org/cryscam/umr_small.wmv) or personal communications. The remaining characteristics were obtained from published data (Deller \& Rupp, 2014).

\begin{tabular}{|c|c|c|c|}
\hline & Acoustic & Robotic & Hand mount \\
\hline Fully automated & Yes & Sometimes & No \\
\hline Time (s per mount) & $0.429 \pm 0.0003$ & $120-240$ & $>60$ \\
\hline Typical mounting media & Micromesh or custom $\dagger$ & Loop or micromesh & Loop or micromesh \\
\hline $\begin{array}{l}\text { Cryoprotectants added to } \\
\text { mounted crystal }\end{array}$ & Yes & Yes & No \\
\hline $\begin{array}{l}\text { Chemical library added to } \\
\text { mounted crystal }\end{array}$ & Yes & No & No \\
\hline Mechanical stress & None & Small & Operator-dependent \\
\hline Specific crystal selection & No & Yes & Yes \\
\hline
\end{tabular}

$\dagger$ Acoustic mounting can deliver specimens to destinations such as direct injection into an X-ray beam (Roessler et al., 2013, 2014). the experimenter. ADE is gentle in that no tools (for example pipette tips) touch the source medium or the destination medium. This prevents contamination, chemical leaching, mechanical stress on crystals or loss of specimen owing to surface adhesion (McDonald et al., 2008). Transfer is fast (500 transfers s ${ }^{-1}$ for multiple transfers to the same micromesh and 2.33 transfers s $^{-1}$ for transfers to different micromeshes), which simplifies serial applications such as distributing crystals onto different micromeshes and combining each crystal with a different chemical. Acoustically transferring both a crystal and a screened chemical and soaking them together on the same micromesh minimizes the use of protein, chemicals and time.

The ejection trajectory is highly accurate, which allows crystals and

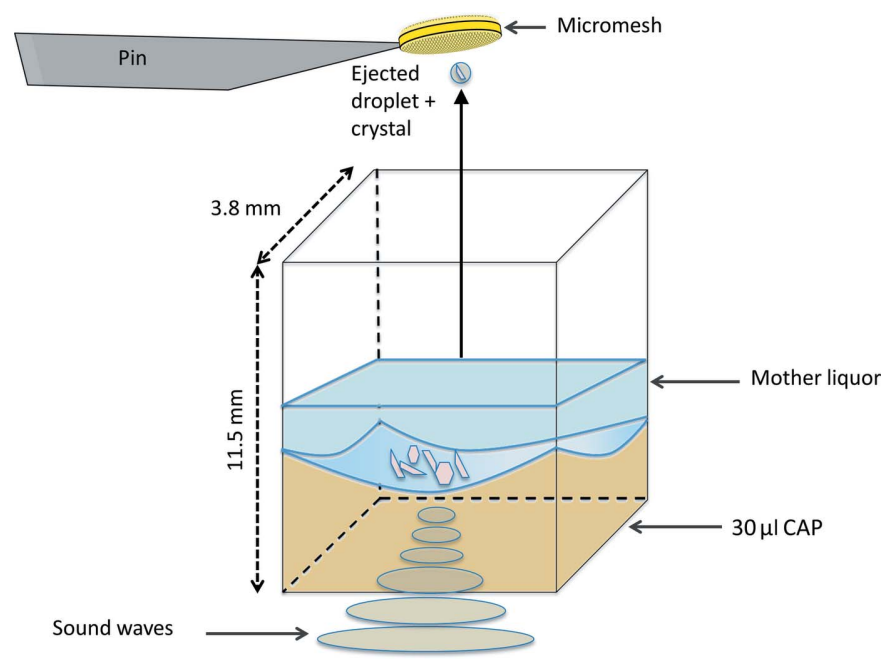

\section{Figure 1}

Acoustic droplet ejection (ADE) from a concave agarose pedestal (CAP). ADE uses sound energy to transfer $2.5 \mathrm{nl}$ microdroplets of liquids (such as chemical libraries) or suspended solids (such as mother liquor containing small protein crystals) from a source well, through a short air column $(1-10 \mathrm{~mm})$ to a micromesh. Sound energy from the transducer is channeled to the focal point (i.e. ejection zone), displacing the surface, where a controlled ejection occurs. The droplet size is governed by the wavelength of the sound emitted; we used a fixed wavelength to eject chemicals and crystals in $2.5 \mathrm{nl}$ increments. Chemicals are ejected from unmodified source wells. Protein crystals are ejected from source wells that have a CAP with the same chemical composition as the mother liquor of the crystals, ensuring that the crystals remain intact and viable for transfer. Agarose, being acoustically transparent, allows the transfer of most suspended solids (such as crystals) with very high precision onto a standard micromesh. Protein crystals in mother liquor are sequestered in the concave basin and suspended above the dead volume. A 2\% agarose solution in the random-coil phase $\left(\right.$ at $\left.100^{\circ} \mathrm{C}\right)$ was mixed in a 1:1 ratio with crystallization conditions for lysozyme, thermolysin, stachydrine demethylase and photosystem II. Wells of a 384-well polypropylene source microplate were overfilled with $70 \mu \mathrm{l}$ of the agarose and precipitant mixture. To create the concave topography of the pedestal, $40 \mu \mathrm{l}$ were removed from the wells after a $3 \mathrm{~s}$ cooling period. screening chemicals to be individually passed from wells in a source plate (described in \$2) through a small (1 mm diameter) aperture and onto a micromesh that is secured in a sealed pin platform box that contains mother liquor (Fig. 2). At present, pin-mounted micromeshes are manually snapped into the pin platform, where they are secured in a fitting that mechanically compresses the metal pin (all components are printed by a three-dimensional printer and print files are available on request). Each micromesh is then individually targeted by our acoustic system, so that crystals and screening chemicals can be transferred from a source plate and combined on the micromesh. The pin platform box ensures that the micromesh is in vapor equilibrium with the mother liquor before, during and after the transfer of crystals and screening chemicals. This means that each crystal can be soaked with its screening chemical on a micromesh for as long as desired without the crystal dehydrating. It is also possible to co-crystallize proteins and chemical fragments (or other screened chemicals) in situ directly on micromeshes using a similar technique (Yin et al., 2014).

This study uses the Echo 550 liquid-handling instrument (Labcyte Inc., Sunnyvale, California, USA) to transfer suspended crystals and chemicals from source wells containing CAPs onto micromeshes. Innovations in the Echo line of instrumentation have decreased the 'dead volume' (an inaccessible region for ejection) at the bottom of each source well to $<4 \mu \mathrm{l}$ (Harris et al., 2008). However, crystallization experiments tend to yield few crystals, many of which then disappear into this $4 \mu \mathrm{l}$ region. Consequently, acoustic crystal transfer is only practical if the crystals are suspended at or near the ejection region.

Here, we describe the use of agarose gels to construct concave pedestals that support protein crystals at a suitable location for acoustic ejection; crystals and chemicals are ejected onto each micromesh for high-throughput screening. 


\section{Table 2}

Time needed for typical serial crystallography applications.

The transfer rate for the Echo 550 is 500 transfers s$^{-1}$ from a single location and 2.33 transfers s ${ }^{-1}$ when moving between source locations or between destination locations. Approximately $1 \mathrm{~min}$ is needed to exchange plates. We assume that there are sufficient pin platform boxes pre-loaded with micromeshes for each experiment. The time to complete the two first tasks was measured ( $\$ 3.2$ and $\$ 3.3$ ), while for the two last tasks it was simulated using water in place of the chemical library (we have not yet acquired a large chemical library).

\begin{tabular}{|c|c|}
\hline Task & Time \\
\hline $\begin{array}{l}\text { Mount crystals onto } 96 \text { micromeshes ( } 50 \mathrm{nl} \text { crystal slurry } \\
\text { dispensed to each micromesh, as described in } \$ 3.2)\end{array}$ & $52 \mathrm{~s}$ \\
\hline $\begin{array}{l}\text { Mount crystals on } 36 \text { micromeshes and combine with heavy- } \\
\text { atom screen }(50 \mathrm{nl} \text { crystal }+5 \mathrm{nl} \text { additive, as described in } \\
\S 3.3)\end{array}$ & $35 \mathrm{~s}$ \\
\hline $\begin{array}{l}\text { Mount crystals on } 96 \text { micromeshes and combine with the } \\
\text { commercial Additive Screen kit ( } 50 \mathrm{nl} \text { crystal }+5 \mathrm{nl} \\
\text { additive) }\end{array}$ & $\sim 1 \min 32 \mathrm{~s}$ \\
\hline $\begin{array}{l}\text { Mount crystals on } 2000 \text { micromeshes and combine with the } \\
\text { fragment library ( } 2000 \text { fragments) }\end{array}$ & $\sim 53 \min 20 \mathrm{~s}$ \\
\hline
\end{tabular}

The concave pedestals consist of acoustically transparent hydrogels (polymerized matrix materials with high water content). The pedestals suspend protein crystals above the dead volume and sequester them precisely at the ejection zone, where the acoustic ejection pulse occurs. Many types of hydrogels are transparent to acoustic energy. We chose agarose to create concave agarose pedestals (CAPs) for this study because agarose is a safe and common laboratory reagent. In contrast, gelatin pedestals require overnight refrigeration and acrylamide pedestals are made with toxic substances.

Crystals can be pipetted onto CAPs for serial transfer onto micromeshes. The pedestal is impermeable to protein crystals but is permeable to mother-liquor chemicals (so the crystals retain the same chemical composition as the mother liquor). Agarose is acoustically transparent, which facilitates easy and rapid serial transfer of crystals from the CAP to micromeshes. In some cases it may be advantageous to serially transfer microcrystals onto micromeshes in this way, both to save time and to minimize the $\mathrm{X}$-ray background contribution from solvent. However, we believe that the largest utility for acoustic crystal mounting will derive from its ability to readily combine just-mounted crystals with chemicals such as heavy atoms, cryoprotectants, additives that improve crystal quality and of course fragment libraries (Table 2). We have also grown crystals directly on CAPs to avoid manual transfer (http:// www.youtube.com/channel/UCtCiMjlzBnq5VYZzrEi3EiQ). When growing crystals directly on CAPs, agarose is a better choice than agar because the impurities in agar cause the matrix to acquire a yellow tint that can make crystals harder to see.

\section{Methods}

We used a commercially available Echo 550 liquid-handling instrument (Labcyte Inc) to transfer two standard crystal samples (lysozyme and thermolysin), a metalloprotein sample (stachydrine demethylase) and membrane-protein crystals (photosystem II) from a 384-well polypropylene microplate (Labcyte Inc) source plate onto pin-mounted micromeshes that were secured in a pin platform box (Fig. 2). The temperature inside the acoustic transfer chamber was tightly controlled at $22^{\circ} \mathrm{C}$. The crystals used in this experiment were selected to represent a broad range of crystallization conditions and physical properties, such as fragile rod-shaped thermolysin, rigid cuboidal lysozyme and plate-shaped stachydrine demethylase crystals. The concave agarose pedestals (CAPs) contained the same chemical environment as the crystal mother liquor, including cryoprotectants. Cryoprotection of lysozyme and stachydrine demethylase was with mother liquor plus $15 \%$ glycerol $(10 \mathrm{ml}$ mother liquor plus $1.5 \mathrm{ml}$ glycerol), thermolysin was soaked in mother liquor plus $20 \%$ ethylene glycol (10 $\mathrm{ml}$ mother liquor plus $2.0 \mathrm{ml}$ ethylene glycol) and photosystem II crystals were stage-soaked to mother liquor plus $30 \%$ glycerol ( $10 \mathrm{ml}$ mother liquor plus 1,2 and $3 \mathrm{ml}$ increasing concentrations of glycerol $)^{\mathbf{1}}$.

To enable the ejection of all crystals, we pre-loaded the source plate with $\sim 30 \mu \mathrm{l}$ CAPs (Fig. 1). Each CAP was composed of $1 \%$ agarose and mother liquor containing cryoprotectant. Each type of protein crystal was separately grown on a cover slip in a standard hanging-drop preparation. The crystals were manually pipetted from their hanging drop onto the CAPs. Each pedestal suspended the crystals above the dead volume that is inaccessible for transfer by the Echo 550 . Furthermore, the concave shape of the pedestal concentrated the crystals in the ejection zone (the middle of each source well). Crystals were acoustically transferred from the CAP onto a pin-mounted micromesh (Fig. 2) and cryocooled for $\mathrm{X}$-ray data collection (cryocooling is described in \$3.3). Subsequent to each crystal-ejection event, the concave shape ensured that the remaining crystals descended to the ejection zone. The concentration of crystals on the CAP determines the average number of crystals ejected with each $50 \mathrm{nl}$ drop (approximately five crystals per micromesh for lysozyme and thermolysin and one crystal per every two micromeshes for stachydrine demethylase and photosystem II; see \$2.3). We used thermolysin crystals to measure the time needed to harvest our specimens. The crystal-harvesting rate was found by averaging 15 timed trials of 495 crystal transfers to five distinct locations on 99 micromeshes, which required an average of $212.4 \mathrm{~s}$ to complete (Table 1).

Since each micromesh contains one or a few crystals, additional chemicals can be acoustically added to the already mounted crystals on the micromesh. For example, chemicals from a fragment library can be rapidly distributed so that one or a few crystals on each micromesh are soaked with each chemical fragment. This system allows easy and fast exploitation of protein crystals for high-throughput screening (or

\footnotetext{
${ }^{1}$ Note that the density of the solution to be ejected by the Echo can be larger than expected. For example, adding $1.5 \mathrm{ml}$ glycerol to $10 \mathrm{ml}$ lysozyme mother liquor only increased the total volume by $0.85 \mathrm{ml}$ ( $43 \%$ less than the added glycerol volume). Glycerol also added less than the expected volume to the mother liquors of stachydrine demethylase (30\% less) and photosystem II (15\% less).
} 
serial crystallography) applications such as fragment library screening, cryo-condition search, heavy-atom screening, crystal improvement with additives and fast screening for diffraction quality.

\subsection{Screening of compatible crystallization conditions}

To demonstrate the general applicability of this crystalmounting method for samples grown using standard crystallization conditions, we surveyed the chemical compatibility of agarose crystal supports with commercial crystallization screens. $15 \mu \mathrm{l}$ of crystallization conditions from 96 deep-well commercial crystallization plates, JBScreen Cryo HTS L (Jena Biosciences), Additive Screen (Hampton Research), MemGold (Molecular Dimensions) and MCSG-4 (Microlytic),

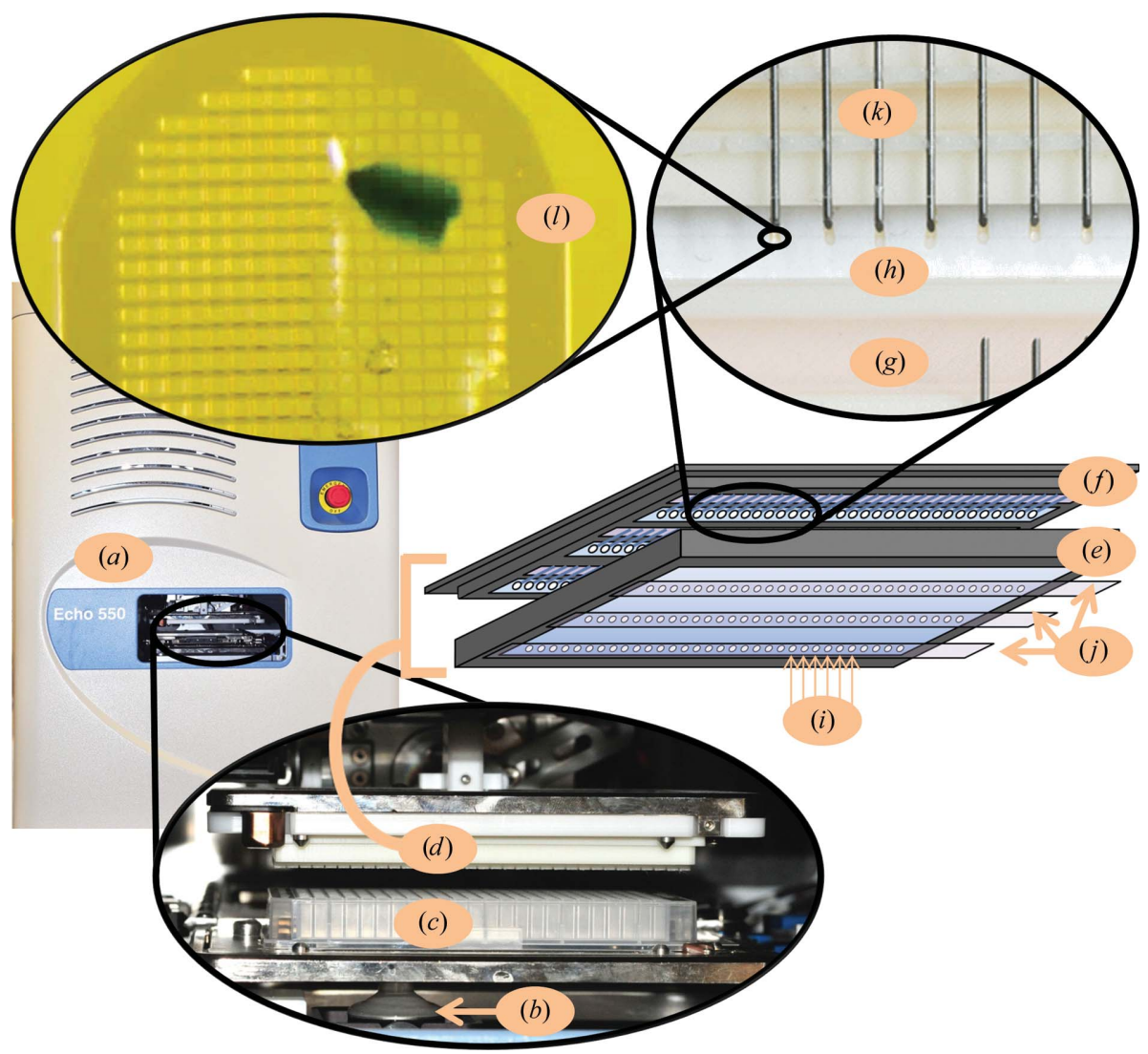

Figure 2

Pin platform box. Crystals and screened chemicals can be transferred in the acoustic transfer chamber of the Echo $550(a)$ using sound pulses generated by a transducer $(b)$ to eject crystals and chemicals contained in a source plate $(c)$ into a pin platform box $(d)$ (shown without the lid for clarity; Yin et al., 2014). The pin platform lid $(e)$ isolates the pin platform $(f)$ to prevent dehydration. The internal environment is governed by mother-liquor solution that is secured in $1 \%$ agarose and is deposited into a moat $(g)$ in the pin platform. The window $(h)$ is used to view specimens and to add components through apertures $(i)$ in the lid. After all of the crystals are mounted, tape is used to seal the apertures $(j)$. The pin platform box contains 96 sockets for securing pin-mounted micromeshes $(k)$. The crystals are transferred onto the pin-mounted micromeshes. Once mounted, the crystals can be combined with cryoprotectants, heavy atoms, crystal-improving additives or with a fragment library; these chemicals are acoustically transferred from the same source plate $(c)$ or from a different source plate. The pin platform box is in equilibrium with the mother liquor before, during and after the crystals and chemicals are transferred onto the micromeshes. The inset $(l)$ shows a magnified view of a photosystem II crystal that was transferred onto the micromesh, where it was combined with a chemical. All components of the pin platform box are three-dimensionally printed (print files are available on request). were dispensed into a 384-well Poly Pro source microplate and trifuged at $1216 \mathrm{~g}$ for $60 \mathrm{~s}$. The volume in each well was keep water out of the of a $2 \%$ solution of agarose in distilled water was prepared in meyer flask and maintained at $\sim 100^{\circ} \mathrm{C}$ on a hotplate agarose dissolved. The agarose solution was then flask with the agarose solution at $70^{\circ} \mathrm{C}$, because higher temperatures lead to bubbles and melt the pipette tips, while lower temperatures cause the concave basin to cool asymmetrically. $15 \mu \mathrm{l}$ of the agarose solution were manually dispensed into each well of the heated 384-well polypropylene plate. Any observed bubbles were ruptured using the pipette tip. The 384well polypropylene plate was removed from the bath and (after cooling) centrifuged (1216 $g$ for $60 \mathrm{~s}$ ). Each CAP was examined for air bubbles, firmness (as verified by probing with a toothpick) and visual evidence of precipitation (owing to incompatibility between mother liquor and agarose). We inspected the concave shape of each CAP. Finally, we added $10 \mu$ l of water to each CAP and attempted to eject droplets of this water using the Echo 550 .

\subsection{Protein crystallization and plate preparation}

A $2 \%$ agarose solution was heated $\left(100^{\circ} \mathrm{C}\right.$ for $\left.\sim 10 \mathrm{~min}\right)$ in a water bath until it reached a random-coil state (a polymer conformation where monomer subunits are randomly oriented but are still bound to adjacent subunits). The agarose solution was then cooled to $70^{\circ} \mathrm{C}$ and mixed in a $1: 1$ ratio with the following mother-liquor solutions: $0.2 \mathrm{M}$ sodium acetate, $8 \% \mathrm{NaCl}$ for lysozyme, $0.05 M \mathrm{NaOH}, 15 \%$ ammonium sulfate for thermolysin, $10 \%$ glycerol, $10 \%$ PEG 3350, $25 \mathrm{~m} M$ hexammine cobalt chloride, $100 \mathrm{~m} M$ HEPES pH 7.0 for stachydrine demethylase and 40\% PEG 5000 for photosystem II.

In order to achieve a concave basin, the wells must be over-filled with tacky agarose (when cooled to $\sim 70^{\circ} \mathrm{C}$, agarose becomes somewhat adhesive) and mother-liquor solution so that the agarose adheres to the walls of the source well, resulting in a bowl-shaped 
Table 3

Crystallization conditions and agarose compatibility screening.

Four commercially available crystallization plates (each containing 96 different conditions) were screened for incompatibility between the components of the commercial kits and $2 \%(w / v)$ agarose. $20 \mu \mathrm{l}$ of each crystallization condition from the commercially available plates were added to the wells of a 384-well polypropylene plate with $20 \mu \mathrm{l} 2 \%$ agarose in the random-coil state. Conditions that resulted in precipitation were recorded. All conditions formed a hardened gel, so this information was not recorded in the table. After cooling, $10 \mu \mathrm{l}$ of water were added to the wells and $2.5 \mathrm{nl}$ drops of this water were ejected using the Echo 550. Wells from which a drop was not ejected were also recorded.

\begin{tabular}{lll}
\hline Commercial crystallization kit & Precipitation & Ejection failure \\
\hline JBScreen Cryo HTS L (Jena Bioscience) & 4 conditions $(4.2 \%)$ & 13 conditions $(13.5 \%)$ \\
Additive Screen (Hampton Research) & 4 conditions $(4.2 \%)$ & 6 conditions $(7 \%)$ \\
MemGold (Molecular Dimensions) & 53 conditions $(55.2 \%)$ & 14 conditions $(15 \%)$ \\
MCSG-4 (Microlytic) & 33 conditions $(34.4 \%)$ & 11 conditions $(11 \%)$ \\
\hline
\end{tabular}

beamlines X12C and X29 at the National Synchrotron Light Source (NSLS). Data sets were processed with HKL-2000 (Otwinowski \& Minor, 2001) and further processed using CTRUN$C A T E$ in the CCP4i suite (Winn et al., 2011). Structures were obtained by molecular substitution from published models and were refined using REFMAC (Winn et al., 2003) and ARP/ $w A R P$ (Perrakis et al., 2001) (starting models: lysozyme, PDB entry 1lyz; thermolysin, 4tln; stachydrine demethylase, 3vca; photosystem II, 1fe1; Diamond, 1974; Holmes \& Matthews,

surface when excess agarose is removed from the center of each well. The wells of a 384-well polypropylene source microplate were overfilled with $70 \mu \mathrm{l}$ of the agarose and mother-liquor mixture using a pipette. After allowing $3 \mathrm{~s}$ for the agarose to adhere to the sides of the well, $40 \mu \mathrm{l}$ were aspirated out of the well from the center. This created a concave basin in the agarose gel (Fig. 1). A custom-made positioning tool secured the pipette tip in the center of each well to ensure a symmetric bowl shape.

Crystals of lysozyme $\left(50 \mathrm{mg} \mathrm{ml}^{-1}\right)$, thermolysin $\left(50 \mathrm{mg} \mathrm{ml}^{-1}\right)$ and stachydrine demethylase $\left(20 \mathrm{mg} \mathrm{ml}^{-1}\right)$ were grown by standard hanging-drop protocols $(4 \mu \mathrm{l}$ of protein solution combined in a 1:1 ratio with mother-liquor solution over a $500 \mu \mathrm{l}$ reservoir). The photosystem II crystals were donated. Crystals were manually pipetted from each hanging drop onto the agarose pedestal, where gravity led them to accumulate in the center. The plate was sealed with adhesive plastic. Using the Echo 550, the supernatant above the crystals was removed in $1 \mu \mathrm{l}$ increments (by serial ejection onto the plastic adhesive that sealed the source plate; no pin platform box was present) until crystals were observed in the ejecta using a light microscope (supernatant removal). A $1 \mu$ l volume was chosen because the emergence of crystals from the CAP was observed to be gradual, so the number of crystals lost in the $1 \mu \mathrm{l}$ supernatant-removal procedure was small compared with the total number in the well. The adhesive plastic was peeled off after the supernatant was removed. $50 \mathrm{nl}$ of crystal suspension was then acoustically transferred from the CAP to each micromesh (Fig. 2). In cases where the crystal concentration was high (lysozyme and thermolysin), each micromesh contained an average of approximately five crystals. In cases where the crystal concentration was low (stachydrine demethylase and photosystem II), only mother liquor was ejected onto some of the micromeshes. If crystals were not observed on each micromesh (using a Leica microscope) then additional transfers were made.

Each micromesh that contained crystals was cryocooled. When cryocooling many crystals on pin-mounted micromeshes, the entire pin platform was manually dropped into liquid nitrogen (see \$3.3). When cryocooling only a few crystals on pin-mounted micromeshes, each crystal was individually cooled by hand. Diffraction data were collected on
1981; Daughtry et al., 2012; Zouni et al., 2001). Each atomic model was further screened for binding to agarose (ZINC database 87496095) using AutoDock Vina (Trott \& Olson, 2010), confirming that the tightest predicted binding pose for agarose monomers has zero electron density ${ }^{2}$ (we could not find any electron density for sugar molecules that might have originated from the agarose gel).

\subsection{Preparing crystals for screening against a heavy-atom library}

Thermolysin and lysozyme crystals were obtained as described in \$2.2. Crystals were manually transferred from the thermolysin and lysozyme hanging drops to a CAP containing thermolysin mother liquor and to a CAP containing lysozyme mother liquor as described in $\$ 2.1$. Additionally, eight watersoluble heavy-atom salt solutions (cupric sulfate, iron chloride, nickel sulfate, hexammine cobalt chloride, potassium iodide, sodium iodide, sodium bromide and copper nitrate) and three insoluble suspensions (platinum chloride, nickel chloride and molybdenum chloride) were added to discrete locations on the same polypropylene source plate. Hence, the same source plate contained all of the building blocks for our screening experiment (the protein crystals and the screened chemicals). To assemble the experiments using these building blocks, two pin platform boxes were loaded with pins and mother liquor (thermolysin mother liquor for the thermolysin crystals and lysozyme mother liquor for the lysozyme crystals; Fig. 2).

\subsection{Assessing the acoustic transparency of hydrogels}

Agarose is one example of a class of materials termed hydrogels, most or all of which we predicted to be functionally transparent to the types of sound waves (frequencies, waveforms etc.) used for acoustic crystal mounting. To determine the acoustic transparency of various hydrogels, three wells of a 384-well polypropylene microplate were prepared with

\footnotetext{
${ }^{2}$ For each protein, AutoDock Vina was used to identify the tightest binding pose of an agarose sugar dimer to the molecular model. Each model was divided into slabs that were long and wide enough to enclose the entire protein and as thick as our computational memory resources allowed. The photosystem II model was a partial model; consequently, AutoDock Vina was only used to search for agarose sugar dimer binding poses in completed areas of the protein
} 


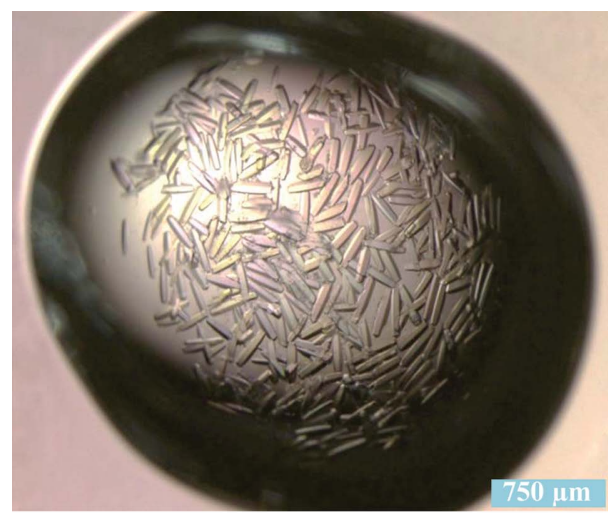

$(a)$

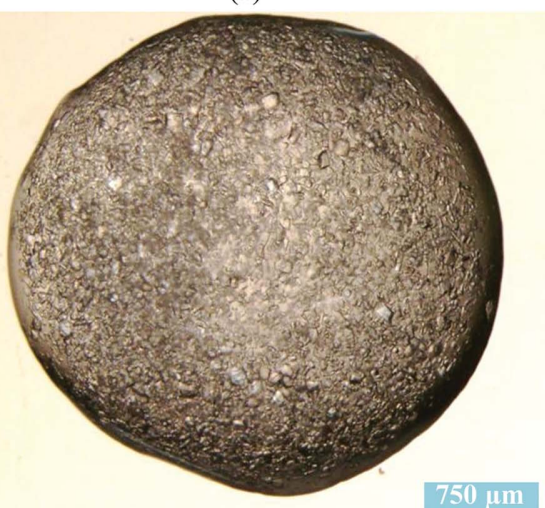

(d)

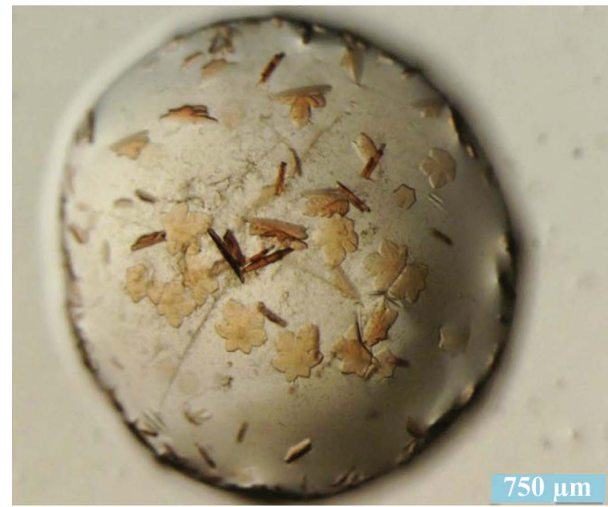

$(g)$

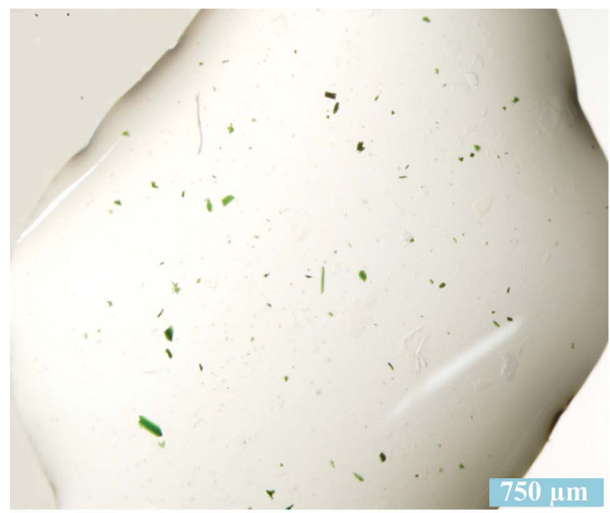

(j)

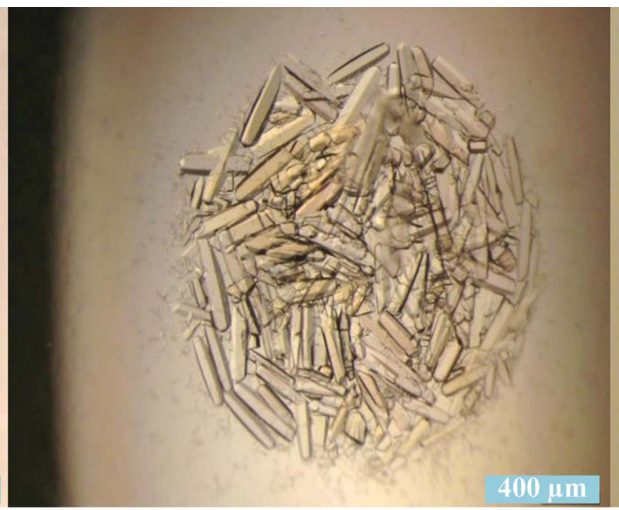

$(b)$

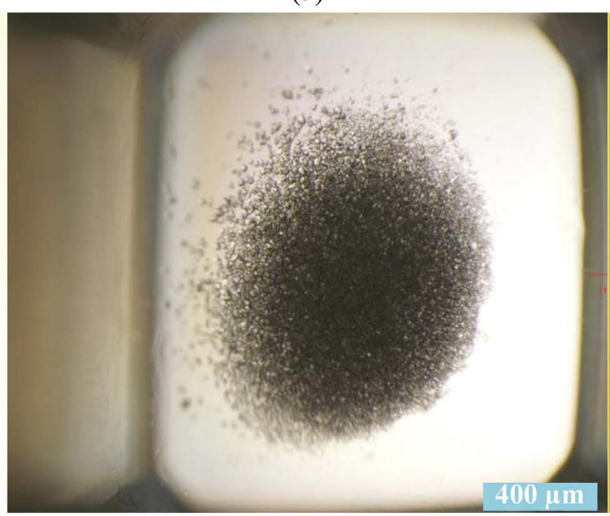

$(e)$

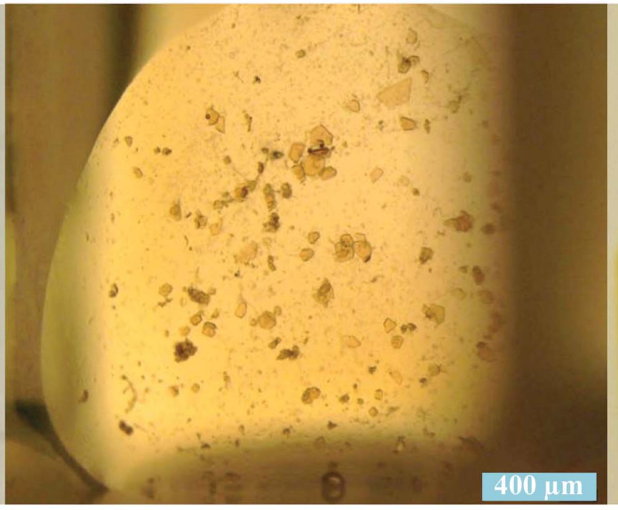

(h)

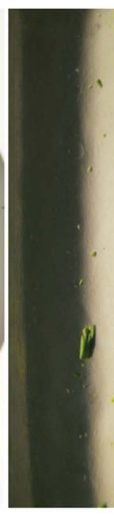

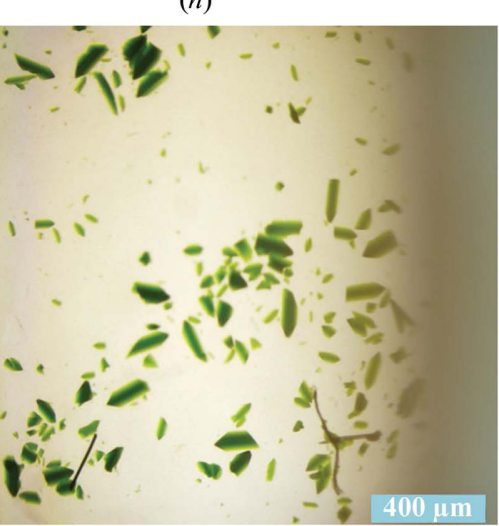

(k)

Figure 3

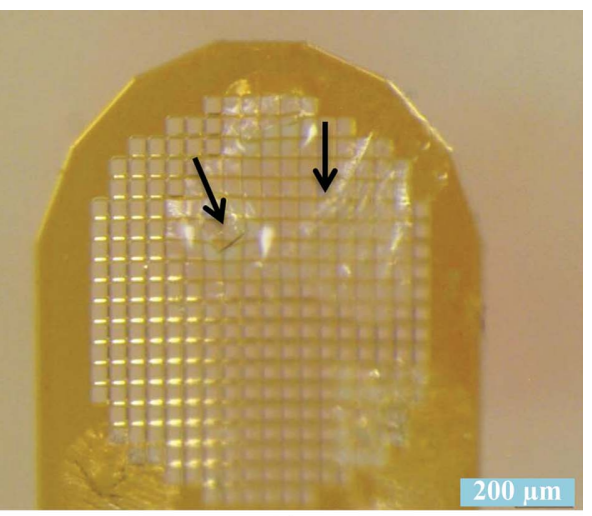

$(c)$

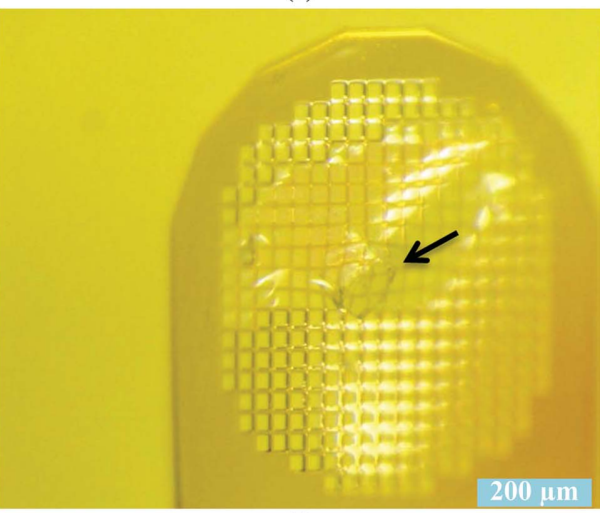

$(f)$

Crystallization, CAP and transfer of thermolysin, lysozyme, stachydrine demethylase and photosystem II. Proteins were crystallized using the hangingdrop method $(a, d, g, j)$. Wells were preloaded with a $1 \%$ agarose and mother-liquor pedestal. Crystals were transferred manually with a pipette into wells of an acoustically transparent 384-well polypropylene plate $(b, e, h, k)$. The concave basin of the CAP caused crystals to concentrate at the ejection zone under the force of gravity. Crystals (indicated by arrows) were transferred onto MiTeGen micromeshes for X-ray diffraction analysis $(c, f, i, l)$ (see Supplementary Fig. S2). 
Table 4

Data-collection and model-refinement statistics.

Diffraction data from acoustically mounted crystals of lysozyme and thermolysin were comparable to diffraction from a manually mounted control crystal (left columns). Diffraction from acoustically mounted crystals of stachydrine demethylase and photosystem II were typical of these crystals (private communication). In the case of lysozyme and thermolysin, each of the ten data sets from acoustically mounted crystals and each of the ten data sets from hand-mounted crystals was obtained from a single crystal. Where appropriate, average values and standard deviations are shown for each group of ten data sets from similar crystals. In the case of stachydrine demethylase and photosystem II, diffraction data from multiple acoustically mounted crystals were combined into a single data set.

\begin{tabular}{|c|c|c|c|c|c|c|}
\hline & \multicolumn{2}{|l|}{ Lysozyme } & \multicolumn{2}{|l|}{ Thermolysin } & Stachydrine demethylase & Photosystem II \\
\hline \multirow{2}{*}{\multicolumn{7}{|c|}{ Crystallization conditions }} \\
\hline & & & & & & \\
\hline Protein $\left(\mathrm{mg} \mathrm{ml}^{-1}\right)$ & \multicolumn{2}{|c|}{50} & \multicolumn{2}{|l|}{50} & 20 & $\mathrm{n} / \mathrm{a}$ \\
\hline Precipitant & \multicolumn{2}{|l|}{$8 \% \mathrm{NaCl}$} & \multicolumn{2}{|c|}{$15 \%$ ammonium sulfate } & $10 \%$ PEG $3350,10 \%$ glycerol & $40 \%$ PEG 5000 \\
\hline Mounting & Acoustic & Hand & Acoustic & Hand & Acoustic & Acoustic \\
\hline $\mathrm{X}$-ray source & NSLS X12C & NSLS X12C & NSLS X25 & NSLS X25 & NSLS X12C & NSLS X29 \\
\hline Wavelength $(\AA)$ & 0.978 & 0.978 & 0.978 & 0.978 & 0.978 & 0.978 \\
\hline Beam width $\times$ height $(\mu \mathrm{m})$ & $150 \times 150$ & $150 \times 150$ & $50 \times 50$ & $50 \times 50$ & $150 \times 150$ & $100 \times 50$ \\
\hline Resolution $(\AA)$ & $1.89 \pm 0.38$ & $2.03 \pm 0.36$ & $1.58 \pm 0.12$ & $1.66 \pm 0.16$ & 3.15 & 4.9 \\
\hline$R_{\text {sym }}$ or $R_{\text {merge }}(\%)$ & $11.75 \pm 3.91$ & $10.94 \pm 4.17$ & $8.3 \pm 3.2$ & $10.9 \pm 4.0$ & 32.7 & 9.4 \\
\hline \multicolumn{7}{|l|}{ Model-refinement statistics } \\
\hline Bond lengths $(\AA)$ & $0.015 \pm 0.010$ & $0.014 \pm 0.010$ & $0.026 \pm 0.003$ & $0.024 \pm 0.004$ & 0.010 & N/A \\
\hline Bond angles $\left({ }^{\circ}\right)$ & $1.68 \pm 0.69$ & $1.60 \pm 0.72$ & $2.34 \pm 0.31$ & $2.19 \pm 0.28$ & 1.470 & N/A \\
\hline
\end{tabular}

pedestals of gelatin ( $3 \%$ unflavored gelatin; commercial gelatin), agarose (2\% agarose; Sigma-Aldrich catalog No. A6877) and acrylamide [16\%(w/v) 29:1 acrylamide; SigmaAldrich catalog No. A7802]. For each hydrogel, we used the Echo 550 WellPing software to send five acoustic pulses $(11.5 \mathrm{MHz})$ through the material and to listen to the resulting reflected acoustic signal. The five reflected acoustic profiles from each material were then averaged.

\section{Results}

\subsection{Agarose pedestals are compatible with most crystallization conditions}

To test the compatibility of agarose pedestals with common crystallization conditions (Table 3 ), $20 \mu \mathrm{l}$ of each crystallization condition was mixed with $20 \mu \mathrm{l} 2 \%$ agarose at $70^{\circ} \mathrm{C}$ and allowed to cool into a gel. Once hardened, each gel was tested for (i) firmness, (ii) acoustic ejection and (iii) the presence of precipitate. $10 \mu \mathrm{l}$ mother liquor was added to the gel and $2.5 \mathrm{nl}$ were ejected out of each well onto a plastic cover using the Echo 550. Transfer success was observed under a Leica microscope. A high percentage of wells were both firm enough to support a distinct layer of mother liquor and able to eject this mother liquor (Table 3). Each well was also examined for precipitation using the Leica microscope. Any solution (agarose and mother liquor) that appeared to form a precipitate was recorded (CAPs were examined with a light microscope and any discoloration was noted as a precipitate). In cases where the initial agarose preparation has a precipitate, adjustment of the agarose concentration and/or the precipitant concentrations usually allowed an effective CAP (data not shown). Crystallization cocktails that stubbornly inhibit gel formation \{for example, ammonium sulfate $\geq$ $30 \%(w / v)[30 \%(w / v)=40 \%$ saturation $]$ or PEG $5000 \geq$ $50 \%(w / v)\}$ and prevent droplet ejection can be soaked in the mother liquor after the gel has hardened. We therefore believe that this method is generally applicable to most common protein crystallization conditions.

\subsection{CAPs eliminate dead volume and reduce loss of crystals}

Lysozyme, thermolysin, stachydrine demethylase and photosystem II crystals were transferred from their hangingdrop crystallization plates (Figs. $3 a, 3 d, 3 g$ and $3 j$, respectively) and suspended on CAPs in a source plate. The concave basin assured that many crystals remained in the ejection zone of the wells (Figs. $3 b, 3 e, 3 h$ and $3 k$ ). After supernatant removal, the crystals on the CAP were acoustically transferred onto micromeshes (Figs. 3c, 3f, $3 i$ and $3 l$ ). Diffraction data from acoustically mounted crystals of lysozyme and thermolysin were comparable to diffraction from manually mounted control crystals, demonstrating that acoustic ejection from a concave agarose pedestal does not adversely affect the quality of the data (Table 4) or of the resulting electron density (see Supplementary Fig. S2). In all cases, the quality of recorded data was compatible with data from manually mounted crystals. AutoDock Vina was used to predict the best binding location between each protein structure and agarose monomers; inspection showed that there was no electron density in these areas. Each electron-density map was also visually examined using Coot (Emsley \& Cowtan, 2004) to verify that 
there was no large contiguous difference density that could correspond to a sugar molecule.

\subsection{Crystals can be combined with chemicals directly on a micromesh}

The thermolysin and lysozyme crystals described in $\$ 2.3$ were transferred onto pin-mounted micromeshes that were secured in two pin platform boxes (as described in §3.2). For each type of protein crystal, $50 \mathrm{nl}$ of crystal suspension were transferred (through apertures) onto each of 36 micromeshes

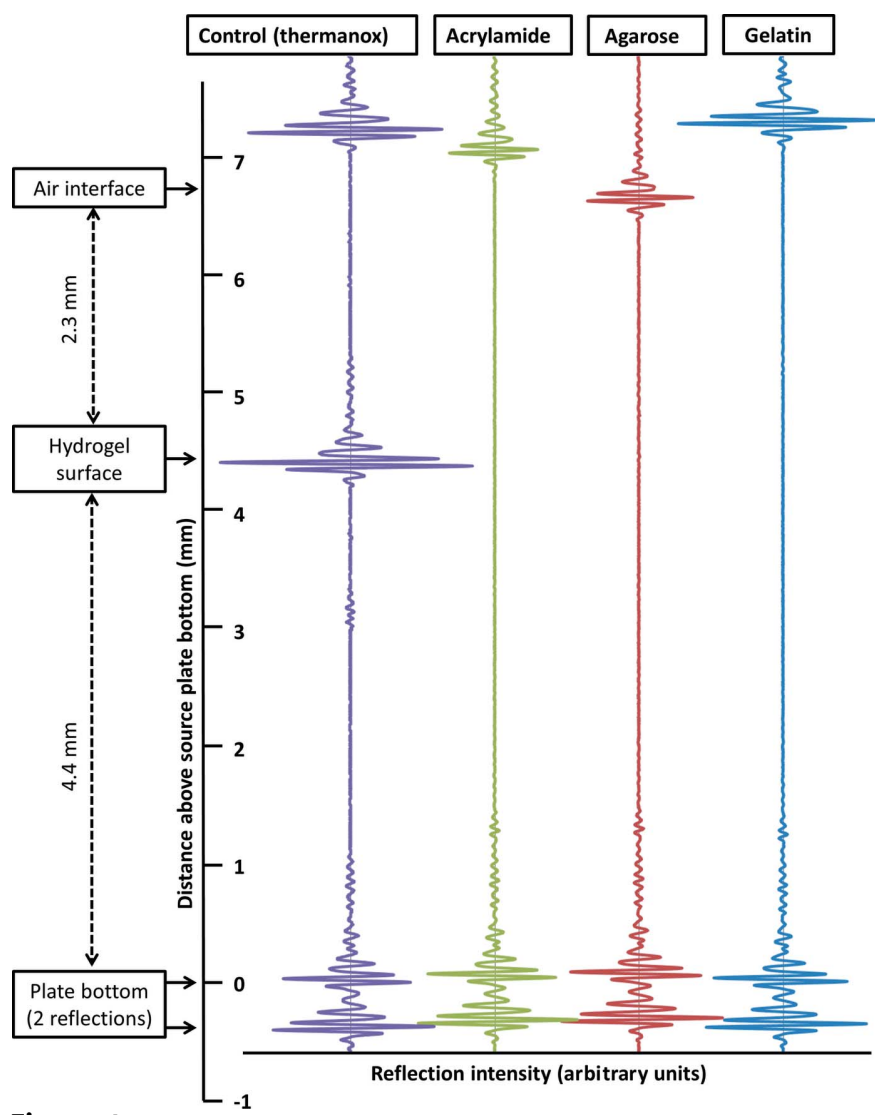

\section{Figure 4}

Acoustic transparency of hydrogels. Many hydrogels are acoustically transparent to the waveform and frequency used to transfer crystals (or other materials) onto X-ray data-collection micromeshes $(11.5 \mathrm{MHz})$. The intensity of the reflected sound is shown for hydrogels of acrylamide (green), agarose (red) and gelatin (blue). In all hydrogel cases, a concave pedestal was deposited to a height of $4.4 \mathrm{~mm}$ in one well of a 384-well polypropylene plate. Water was then added to a height of $6.7 \mathrm{~mm}$. Five acoustic pings were then transmitted through each well using the Echo 550 and the reflected intensities were recorded as a function of time. The five pulses were averaged for each substance and the averaged values were plotted on a single graph; the horizontal axis is the measured reflected intensity (arbitrary units) and the vertical axis is time. In our control (purple), a Thermanox cover slip was placed on an agarose support to show an example of a material that is acoustically semitransparent (see Supplementary Fig. S1). Because the speed of sound in all of these substances is virtually identical to that in water, the vertical axis is displayed as a distance (in millimetres). The expected location of the interface between the hydrogel and the water is indicated. Acoustic transfer of crystals from a support matrix to micromeshes can only occur if the largest reflection is from the air-water interface. In the case of the three classes of hydrogels tested, the observed acoustic reflection from the gel-water interface was zero, indicating that all of these materials are possible candidates for positioning specimens at the acoustic focus point. (on average each micromesh contained approximately five crystals). Once all of the crystals were distributed to micromeshes, each heavy-atom solution described in $\$ 2.3$ was acoustically transferred (through apertures) onto three different crystal-containing micromeshes of thermolysin and three of lysozyme. Three controls with no heavy atoms were included for each type of protein crystal. Each micromesh with crystals plus heavy atoms was soaked for $1 \mathrm{~h}$. The two pin platform boxes were in equilibrium with the mother liquors of thermolysin and lysozyme (Fig. 2), so the crystals were soaked without dehydrating. After soaking, the adhesive tape was detached from the back of each pin platform and the lid was removed. Each pin platform (filled with crystal-containing micromeshes) was dropped 'face down' into liquid nitrogen, so that the cryocoolant flowed through the window of the pin platform and flash-cooled each of the crystals. Under liquid nitrogen, the pin platform was rotated to face up and each pinmounted micromesh was manually inserted into a MiTeGen Reusable Base (model B1A-R) ${ }^{4}$.

$\mathrm{X}$-ray data were obtained from all 36 thermolysin heavyatom soaks and from all 36 lysozyme heavy-atom soaks. The data revealed anomalous signal for some known lysozymebinding heavy atoms (nickel sulfate and the iodide salts) but not for sodium bromide (which is a lysozyme ligand in a Protein Data Bank structure). Surprisingly, copper sulfate also yielded a detectable anomalous signal when soaked with lysozyme (the PDB did not previously contain a copper derivative of lysozyme). The structure of this derivative was readily solved (PDB entry 4p2e) using the anomalous diffraction from three bound $\mathrm{Cu}$ atoms (one at a twofold position near Leu129, another coordinated by His15 and Glu7, and a third discreetly disordered copper near Asp52; similar to Teichberg et al., 1974). None of the insoluble salts yielded anomalous data when soaked with lysozyme or thermolysin. For both thermolysin and lysozyme, all of the heavy atoms with accessible white lines (excluding iodine and iron) were confirmed to have been transferred by observing a fluorescence peak at the expected energy using a monochromator excitation scan. Table 2 summarizes the time needed for the Echo 550 to perform soaking experiments of this type.

\subsection{Hydrogels are acoustically transparent}

This study reports the use of agarose gels to support protein crystals at a suitable location for automatic crystal transfer using ADE. Since hydrogels are composed principally of water, we hypothesized that they are likely to be acoustically transparent. Other materials tested for acoustic transparency include gelatin and cross-linked polyacrylamide gels. All of the tested hydrogels were shown to be completely acoustically

\footnotetext{
${ }^{4}$ We have acquired a Staübli six-axis robot (model TX60) to automate the cryocooling of pin-mounted micromeshes from a pin platform box into a robotic V1 uni-puck. The system was not used for the crystals described here because it was not operational when this work was performed. The design for this system is illustrated in Supplementary Fig. S3. A prototype for the robotic system to transfer pin-mounted micromeshes (with crystals on them) into MiTeGen Reusable Bases and onto a conventional puck lid is shown in Supplementary Fig. S4..
} 
transparent (Fig. 4). Cross-linked acrylamide (green) demonstrated no visible reflection at the gel-water interface, but did show noticeable attenuation in the reflected intensity at the water-air interface. This may indicate that some scattering or absorption occurred in the body of the gel, although no reflection was visible at the surface. The scattering/absorption from the $2 \%$ agarose gel was much smaller (red), but like the acrylamide there was zero reflection from the gel-water interface. The gelatin (blue) showed no attenuation of the surface reflection and no reflection from the gel-liquid interface. Agarose was selected for this study because it is a common laboratory material, it hardens faster than gelatin and it is safer to work with than acrylamide. However, both gelatin and polyacrylamide were found to be suitable supports for protein crystal transfer using acoustic methods (data not shown). Noncrystallographic applications that could benefit from acoustic touch-less ultralow-volume specimen preparation (such as SAXS and electron microscopy) may be incompatible with agarose supports. In cases where the properties of agarose are found to be unsuitable, other hydrogels may offer an acoustically compatible solution. In cases where the objective is not to eject crystals but rather to monitor crystal growth, an acoustically semi-transparent medium such as Thermanox may be suitable. Recently, $1 \%(w / v)$ agar was used to fabricate a coupling 'plug' that conducted sound energy from an acoustic transducer to a crystal suspension at the Linear Coherent Light Source (LCLS; Roessler et al., 2014). The sound pulses were used to inject crystal containing droplets into the LCLS at a rate of 60 crystal injections per second, matching the LCLS pulse frequency in order to achieve a $60 \%$ 'hit rate' of X-ray pulses that yielded diffraction patterns.

\section{Discussion}

Full automation of the high-throughput macromolecular crystal structure determination pipeline would increase productivity in conventional structural biology, as well as enable novel discovery-based solutions to stubborn problems in structural biology (particularly using high-throughput screening of chemical libraries). This goal has been frustrated by the difficulty involved in automating fast transfer of crystals from growth plates onto supports suitable for X-ray data collection. In cases where very high speed is not required, robotic solutions (Viola et al., 2007), laser tweezer-assisted mounting (Wagner et al., 2013) and laser-assisted recovery on thin films (Cipriani et al., 2012) are promising alternatives to manual mounting of individual crystals. For fast serial mounting of crystals of a particular protein, investing time to prepare a CAP allows rapid mounting using acoustic methods. We have demonstrated that acoustic crystal mounting from CAPs will sustain a high rate of 2.33 transfers $\mathrm{s}^{-1}$. Combined with automated protein production (Banci et al., 2006; Gräslund et al., 2008), crystallization (Bolanos-Garcia \& Chayen, 2009) and end-station automation (Snell et al., 2004), this will accelerate the output of crystallization facilities to match the data-collection speeds available at next-generation synchrotrons.

Presently, acoustic transfer technology is an advanced method for small-volume liquid transfer. Compared with conventional methods, the acoustic transfer method does not require high-level hand coordination or dexterity. Automated crystal mounting at speeds of several transfers per second prevents loss of crystal viability owing to desiccation, and allows the crystals to be soaked in crystallo (on a micromesh) with chemical libraries such as chemical fragments, heavy atoms, cryoprotectants etc. Acoustic ejection also eliminates contact between specimens and pins, tips and nozzles, which reduces the risk of cross-contamination with laboratory compounds and contamination by chemicals that leach out of the plastic tubing (McDonald et al., 2008).

Acoustic micro-mounting with no dead volume (and no lost volume per transfer) is particularly advantageous when purified protein is in limited supply. Advances in protein expression and purification have significantly relaxed the sourcematerial bottleneck in crystallography, but stubborn cases with poorly expressing proteins still occur. Acoustic ejection of protein crystals from CAPs saves scarce purified protein resources by ensuring that all or most of the available protein crystals are rapidly dispensed to micromeshes, where each can be individually combined with chemicals in a high-throughput manner. Acoustic transfer also economizes on chemicals, such as fragment libraries, which are difficult to obtain in large quantities. Thus, acoustic transfer from CAPs allows highthroughput screening of chemical libraries even in cases of crystals of poorly expressing proteins.

Acoustic crystal handling accelerates the rate of specimen preparation to match the rate at which specimens might be examined at modern synchrotron X-ray sources. Automation also has other advantages in addition to speed. A fully automated structure-determination pipeline (including crystal handling) allows a researcher with no laboratory access to orchestrate cutting-edge science by linking the capabilities of automated protein production and purification, automated crystal growth and automated crystal handling and data collection. Full automation will also preserve the intact flow of machine-generated metadata for the full project lifecycle. Most importantly, the automation of specimen handling will make available to all researchers the utility of centrally archived chemical libraries (including fragment libraries, heavy atoms, crystal-improving additives and cryoconditions) because the Echo 550 will be located at a central facility so that chemical acquisition costs can be pooled among a community of users.

Using the strategies outlined here, high-throughput screening can be accomplished rapidly and using limited quantities of protein and chemicals. By sequestering crystals into the ejection zone in a concave basin, most of the crystals in the well can be ejected onto micromeshes. Pre-loaded concave agarose pedestals simplify acoustic crystal transfer and increase yields for easy access to serial crystallography techniques such as ligand screening, cryo-search, heavy-atom screening and crystal improvement. 
Personnel for this study were recruited largely through the 2013 spring and summer session and 2014 spring session of the Science Undergraduate Laboratory Internships Program (SULI) and Graduate Research Internship Program (GRIP) supported through the US Department of Energy, Office of Science, Office of Workforce Development for Teachers and Scientists (WDTS). Major ongoing financial support for acoustic droplet ejection applications was through the Brookhaven National Laboratory/US Department of Energy, Laboratory Directed Research and Development Grant 11-008 and from the Offices of Biological and Environmental Research and of Basic Energy Sciences of the US Department of Energy, and from the National Center for Research Resources (P41RR012408) and the National Institute of General Medical Sciences (P41GM103473) of the National Institutes of Health. Additional support was provided by a Louis Stokes Alliances for Minority Participation fellowship. Data for this study were measured on beamlines X12C, X25 and X29 of the National Synchrotron Light Source. We thank Jan Kern and Rosalie Tran for kindly supplying photosystem II crystals and supporting our efforts to determine that these specimens are suitable for serial crystallography with acoustic methods. We thank Labcyte Inc., and particularly Joe Olechno, Richard Stearns and Richard Ellson, for their support and guidance. We thank the coeditor and the reviewers of the manuscript for taking the time to help us to address areas that were outside our expertise. Author contributions: ASS designed the experiment and wrote the paper. CMC, DLE, AS, CGR, ET, OC and ASS grew crystals, obtained data and analyzed data. ASS and KJ designed and built the labware. ASS, CGR and RMS trained and supervised student interns. RA and AMO provided the expressed protein. MA, CGR, AMO and ASS designed a related fragment-screening system that supports the current effort.

\section{References}

Banci, L. et al. (2006). Acta Cryst. D62, 1208-1217.

Bolanos-Garcia, V. M. \& Chayen, N. E. (2009). Prog. Biophys. Mol. Biol. 101, 3-12.

Cipriani, F., Röwer, M., Landret, C., Zander, U., Felisaz, F. \& Márquez, J. A. (2012). Acta Cryst. D68, 1393-1399.

Daughtry, K. D., Xiao, Y., Stoner-Ma, D., Cho, E., Orville, A. M., Liu, P. \& Allen, K. N. (2012). J. Am. Chem. Soc. 134, 2823-2834.

Deller, M. C. \& Rupp, B. (2014). Acta Cryst. F70, 133-155.

Diamond, R. (1974). J. Mol. Biol. 82, 371-391.
Ellson, R., Mutz, M., Browning, B., Lee, L., Miller, M. F. \& Papen, R. (2003). J. Assoc. Lab. Autom. 8, 29-34.

Emsley, P. \& Cowtan, K. (2004). Acta Cryst. D60, 2126-2132.

Gräslund, S. et al. (2008). Nature Methods, 5, 135-146.

Harris, D., Mutz, M., Sonntag, M., Stearns, R., Shieh, J., Pickett, S., Ellson, R. \& Olechno, J. (2008). J. Assoc. Lab. Autom. 13, 97-102.

Holmes, M. A. \& Matthews, B. W. (1981). Biochemistry, 20, 69126920.

Maire, A. le, Gelin, M., Pochet, S., Hoh, F., Pirocchi, M., Guichou, J.-F., Ferrer, J.-L. \& Labesse, G. (2011). Acta Cryst. D67, 747-755.

McDonald, G. R., Hudson, A. L., Dunn, S. M., You, H., Baker, G. B., Whittal, R. M., Martin, J. W., Jha, A., Edmondson, D. E. \& Holt, A. (2008). Science, 322, 917.

Otwinowski, Z. \& Minor, W. (2001). International Tables for Crystallography, Vol. F, edited by M. G. Rossmann \& E. Arnold, pp. 226-235. Dordrecht: Kluwer.

Perrakis, A., Harkiolaki, M., Wilson, K. S. \& Lamzin, V. S. (2001). Acta Cryst. D57, 1445-1450.

Roessler, C. G., Kuczewski, A., Stearns, R., Ellson, R., Olechno, J., Orville, A. M., Allaire, M., Soares, A. S. \& Héroux, A. (2013). J. Synchrotron Rad. 20, 805-808.

Roessler, C. G., Allaire, M., Orville, A. M., Soares, A. S. (2014). Acta Cryst. A70, C1156.

Snell, G., Cork, C., Nordmeyer, R., Cornell, E., Meigs, G., Yegian, D., Jaklevic, J., Jin, J., Stevens, R. C. \& Earnest, T. (2004). Structure, 12, 537-545.

Soares, A. S., Engel, M. A., Stearns, R., Datwani, S., Olechno, J., Ellson, R., Skinner, J. M., Allaire, M. \& Orville, A. M. (2011). Biochemistry, 50, 4399-4401.

Teichberg, V. I., Sharon, N., Moult, J., Smilansky, A. \& Yonath, A. (1974). J. Mol. Biol. 87, 357-368.

Trott, O. \& Olson, A. J. (2010). J. Comput. Chem. 31, 455-461.

Villaseñor, A. G., Wong, A., Shao, A., Garg, A., Donohue, T. J., Kuglstatter, A. \& Harris, S. F. (2012). Acta Cryst. D68, 893-900.

Villaseñor, A. G., Wong, A., Shao, A., Garg, A., Kuglstatter, A. \& Harris, S. F. (2010). Acta Cryst. D66, 568-576.

Viola, R., Carman, P., Walsh, J., Miller, E., Benning, M., Frankel, D., McPherson, A., Cudney, B. \& Rupp, B. (2007). J. Appl. Cryst. 40, 539-545.

Viola, R., Walsh, J., Melka, A., Womack, W., Murphy, S., RiboldiTunnicliffe, A. \& Rupp, B. (2011). J. Struct. Funct. Genomics, 12, 77-82.

Wagner, A., Duman, R., Stevens, B. \& Ward, A. (2013). Acta Cryst. D69, 1297-1302.

Winn, M. D. et al. (2011). Acta Cryst. D67, 235-242.

Winn, M. D., Murshudov, G. N. \& Papiz, M. Z. (2003). Methods Enzymol. 374, 300-321.

Yin, X., Scalia, A., Leroy, L., Cuttitta, C. M., Polizzo, G. M., Ericson, D. L., Roessler, C. G., Campos, O., Ma, M. Y., Agarwal, R., Jackimowicz, R., Allaire, M., Orville, A. M., Sweet, R. M. \& Soares, A. S. (2014). Acta Cryst. D70, 1177-1189.

Zouni, A., Witt, H. T., Kern, J., Fromme, P., Krauss, N., Saenger, W. \& Orth, P. (2001). Nature (London), 409, 739-743. 